\title{
System 3 diagnostic process: the lateral approach
}

This article was published in the following Dove Press journal:

International Journal of General Medicine

16 October 2012

Number of times this article has been viewed

\section{Taro Shimizu' \\ Yasuharu Tokuda² \\ 'Rollins School of Public Health, Emory University, Atlanta, GA, USA; ${ }^{2}$ Institute of Clinical Medicine, Graduate School of Comprehensive Human Sciences, University of Tsukuba, Ibaraki, Japan}

Correspondence: Taro Shimizu Rollins School of Public Health, Emory University, Atlanta, GA, USA

Email shimizutaro7@gmail.com

\begin{abstract}
The process of obtaining diagnosis is described as a dual-process model, including the intuitive process, and the analytical process. The similarity between the two systems is that they both infer a diagnosis from patient-derived information. Here we present another process by which to elicit the diagnosis: asking direct questions of the patient themselves, such as "What do you think is the cause?" or "What do you suspect is wrong?" This simple method would enable us to elicit pivotal information for diagnosis. Asking patients direct questions allows them to think about the cause of their own problem and suggest their own diagnosis. This method of reasoning is completely different from the two above-mentioned systems and may represent a third approach. We highlight this third process as an important strategy, thereby using this third effective method of inquiry to facilitate quick and effective diagnosis in conjunction with former two systems.
\end{abstract}

Keywords: diagnosis, diagnostic process, clinical problem solving, dual-process model, clinical reasoning

The process of diagnosis is described as a dual-process model. One is the intuitive process, which usually enables clinicians to make a quick and artistic diagnosis (system 1), and the other is the analytical process which places more emphasis on a comprehensive and scientific diagnostic approach (system 2 ). ${ }^{1}$ As an analogy, using the intuitive process, a mountain climber seeks to reach the peak by the shortest route, whereas in the analytical process, the climber takes a less direct but well paved roadway. The similarity between the two systems is that they both infer a diagnosis from patient-derived information. Consider a case of a 52-year-old male patient presenting at the emergency room with intense left lower abdominal pain developed at midnight. The patient's hidden concerns are recognized by asking, "What do you think is the cause?" or "What do you suspect is wrong?". The patient responds, "It must be kidney stones. I have suffered from them many times before." In this case, the likelihood of a diagnosis of urolithiasis is very high. By simply questioning the patient with questions, such as "Why?" or "How did this start?", we are able to elicit information pivotal to the diagnosis. Asking patients allows them to think about the cause of their problem and suggest their own diagnosis. This method of reasoning is completely different from the two abovementioned systems, and may represent a third approach. In the former two processes, physicians undertake diagnostic reasoning based on the constellation of patient information. However, in the third process, named by us as system 3, the diagnosis in the case above is suggested by the patient, not by the physician. Compared with other two systems, this process may involve lateral thinking. ${ }^{2}$ In the mountain-climbing analogy, the system 
3 approach would involve reaching the top of the mountain using a helicopter. This lateral approach has been sporadically mentioned in various forms in the medical field. ${ }^{3,4}$ Similar to the intuitive and analytical processes, system 3 , the lateral process, has its advantages and disadvantages. The main disadvantage involves anchoring bias due to overdependence on the thoughts of the patient. Proposing system 3 is not merely for the purpose of classification. We highlight this third process as an important strategy in making a diagnosis, thereby using this third effective method of enquiry to facilitate quick and effective diagnosis in conjunction with the former two systems.

\section{Disclosure}

The authors report no conflicts of interest in this work.

\section{References}

1. Norman G. Dual processing and diagnostic errors. Adv Health Sci Educ Theory Pract. 2009;14 Suppl 1:37-49.

2. De Bono E. Lateral Thinking: Creativity Step by Step. New York, NY: Harper and Row; 1970.

3. Hernandez JS, Varkey P. Vertical versus lateral thinking. Physician Exec. 2008;34:26-28.

4. Ho AM, Ricci CJ, Ng CS, et al. The medial-transverse approach for internal jugular vein cannulation: an example of lateral thinking. J Emerg Med. 2012;42(2):174-177.

\section{Publish your work in this journal}

The International Journal of General Medicine is an international, peer-reviewed open-access journal that focuses on general and internal medicine, pathogenesis, epidemiology, diagnosis, monitoring and treatment protocols. The journal is characterized by the rapid reporting of reviews, original research and clinical studies across all disease areas.
A key focus is the elucidation of disease processes and management protocols resulting in improved outcomes for the patient. The manuscript management system is completely online and includes a very quick and fair peer-review system. Visit http://www.dovepress.com/ testimonials.php to read real quotes from published authors. 